\title{
A gain scheduling control of nonlinear systems along a reference trajectory
}

\author{
Atsushi Fujimori, Svante Gunnarsson, Mikael Norrlöf \\ Division of Automatic Control \\ Department of Electrical Engineering \\ Linköpings universitet, SE-581 83 Linköping, Sweden \\ WWW: http://www. control.isy.liu.se \\ E-mail: fujimori@isy.liu.se, svante@isy.liu.se, \\ mino@isy.liu.se
}

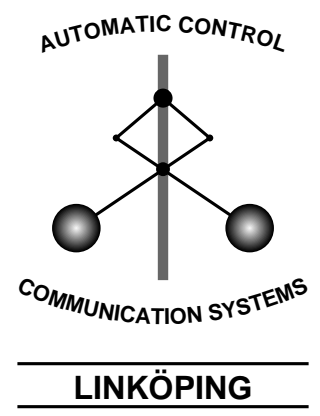

Report no.: LiTH-ISY-R-2654

Submitted to 16th IFAC World Congress

Technical reports from the Control \& Communication group in Linköping are available at http://www.control.isy.liu.se/publications. 


\begin{abstract}
This paper presents a gain scheduling control of a nonlinear system in which the reference trajectory is given in advance. Multiple frozen operating times are chosen on the reference trajectory and a linear time invariant model is obtained at each operating time. A linear parameter varying model is then constructed by interpolating the region between the neighbor frozen operating times. A gain scheduling state feedback law is designed by a linear matrix inequality formulation. The effectiveness is demonstrated in a numerical simulation of a tracking control of a two-link robot arm.
\end{abstract}

Keywords: Nonlinear control, linearization, tracking systems, Lyapunov function, robot arms 


\title{
A GAIN SCHEDULING CONTROL OF NONLINEAR SYSTEMS ALONG A REFERENCE TRAJECTORY
}

\author{
Atsushi Fujimori* $^{*}$ Svante Gunnarsson ${ }^{\dagger}$ and Mikael Norrlöf ${ }^{\dagger}$ \\ ${ }^{*}$ Department of Mechanical Engineering, Shizuoka University \\ 3-5-1 Johoku, Hamamatsu 432-8561, Japan \\ Email: tmafuji@ipc.shizuoka.ac.jp \\ ${ }^{\dagger}$ Department of Electrical Engineering, Linköping University \\ SE-581 83, Linköping, Sweden
}

\begin{abstract}
This paper presents a gain scheduling control of a nonlinear system in which the reference trajectory is given in advance. Multiple frozen operating times are chosen on the reference trajectory and a linear time invariant model is obtained at each operating time. A linear parameter varying model is then constructed by interpolating the region between the neighbor frozen operating times. A gain scheduling state feedback law is designed by a linear matrix inequality formulation. The effectiveness is demonstrated in a numerical simulation of a tracking control of a two-link robot arm. Copyright (c)2000 IFAC
\end{abstract}

Keywords: Nonlinear control, linearization, tracking systems, Lyapunov function, robot arms.

\section{INTRODUCTION}

The robust control techniques (McFarlane and K. Glover, 1990; Zhou, et al., 1996) take into consideration uncertainties due to linearization and/or unmodeled factors to achieve the stability and performance of the closed loop system; that is, the control system is designed in order to satisfy robust stability and/or robust performance conditions. However, if the uncertainties are beyond a level, the controllers designed by the robust control techniques no longer satisfy the stability and the specified performance. Gain scheduling is one of the candidates to control nonlinear systems whose dynamics changes with different operating conditions (Leith and Leithead, 2000; Rugh and Shamma, 2000). One of the gain scheduling techniques for nonlinear systems is based on Takagi-Sugeno (T-S) fuzzy model (Takagi and Sugeno, 1985; Palm, et al., 1997; Tang, et al., 1998; Hsieh, et al., 2001). In this method, fuzzy logic is used to construct a T-S model which approximates the behavior of the nonlinear system (Takagi and Sugeno, 1985). A difficulty in this method is how to generate proper fuzzy rules according to the nonlinear behavior. For example, in a tracking control problem of a two-link robot arm, Palm et al. (1997) prepared thirty-six fuzzy rules to construct a T-S model, while Tang et al. (1998) and Hsieh et al. (2001) applied the genetic algorithm (GA) to optimize the fuzzy rules and the membership functions. These techniques required a great effort to obtain a feasible T-S model because a number of the GA parameters had to be 
decided. In tracking problems of nonlinear systems, when a reference trajectory is generally given in advance, it is not necessary to construct a model which represents a complete behavior of the nonlinear system but it is enough to model the behaviors of the nonlinear system near the reference trajectory.

This paper presents a gain scheduling technique for nonlinear systems whose reference trajectory is given in advance. Multiple frozen operating times are chosen on the reference trajectory and a linear time invariant (LTI) model is obtained at each frozen operating time. Then, a linear parameter varying (LPV) model is constructed by interpolating the region between the neighbor frozen operating times. This modeling avoids the tedious process for generating fuzzy rules (Palm, et al., 1997; Tang, et al., 1998; Hsieh, et al., 2001). Using the interpolative LPV model, a gain scheduling state feedback (GS-SF) law is designed by a linear matrix inequality (LMI) formulation. The effectiveness of the proposed method is demonstrated in a numerical simulation of a tracking control of a twolink robot arm (Palm, et al., 1997; Hsieh, et al., 2001).

\section{INTERPOLATED LPV MODEL}

Let us consider a nonlinear autonomous system given by

$$
\dot{x}(t)=f(x(t), u(t))
$$

where $u(t)$ and $x(t)$ are $m$-dimensional input and $n$ dimensional state vectors, respectively. In this paper, it is assumed that $x(t)$ is available for feedback. Let $x^{d}(t)$ and $u^{d}(t)$ be a known state reference trajectory and a corresponding reference input, respectively. The objective of control is to track the reference trajectory $x^{d}(t)$ as close as possible. Defining the deviation variables $\delta x(t) \triangleq x(t)-x^{d}(t)$ and $\delta u(t) \triangleq u(t)-$ $u^{d}(t)$, the tracking control problem for the nonlinear autonomous system Eq. (1) is transformed into the stabilization problem for the nonlinear nonautonomous system

$$
\delta \dot{x}(t)=f(x(t), u(t))-f\left(x^{d}(t), u^{d}(t)\right)
$$

That is, the objective is to regulate $\delta x(t)$ to zero; $\delta x(t) \rightarrow$ $0\left(x(t) \rightarrow x^{d}(t)\right)$. Note that $\dot{x}^{d}(t)$ is not necessarily zero. Since the reference trajectory $\left(x^{d}(t), u^{d}(t)\right)$ is given in advance, it is sufficient to take into consideration the dynamics governed by Eq. (1) near the reference trajectory. Expanding $f(x, u)$ at $(x, u)=\left(x^{d}, u^{d}\right)$ and approximating it by the constant and the first deriva- tive terms, Eq. (1) is written as

$$
\delta \dot{x}(t)=A\left(x^{d}, u^{d}\right) \delta x(t)+B\left(x^{d}, u^{d}\right) \delta u(t)
$$

where

$$
\begin{aligned}
& \left.A\left(x^{d}, u^{d}\right) \triangleq \frac{\partial f(x, u)}{\partial x^{T}}\right|_{\left(x^{d}, u^{d}\right)}, \\
& \left.B\left(x^{d}, u^{d}\right) \triangleq \frac{\partial f(x, u)}{\partial u^{T}}\right|_{\left(x^{d}, u^{d}\right)}
\end{aligned}
$$

Let $\tau$ be the operating time which identifies the reference trajectory. It is supposed that the reference trajectory $\left(x^{d}(\tau), u^{d}(\tau)\right)$ is given during the operating range $\tau \in\left[0, \tau_{e}\right]$, where $\tau_{e}$ is the terminal time of $\tau$. When operating the control system, $\tau$ is essentially the same as $t$. It is however convenient that $\tau$ is distinguished from $t$ and is considered as a varying parameter for constructing an LPV model. Let us choose $r$ frozen operating times during the operating range as

$$
0 \leq \tau_{1}<\cdots<\tau_{r} \leq \tau_{e}
$$

Then, for these frozen times, LTI models are obtained as

$$
\begin{gathered}
\delta \dot{x}(t)=A_{i} \delta x(t)+B_{i} \delta u(t) \\
A_{i} \triangleq A\left(x^{d}\left(\tau_{i}\right), u^{d}\left(\tau_{i}\right)\right), \\
B_{i} \triangleq B\left(x^{d}\left(\tau_{i}\right), u^{d}\left(\tau_{i}\right)\right) \\
(i=1, \cdots, r)
\end{gathered}
$$

These LTI models are valid near $\left(x^{d}\left(\tau_{i}\right), u^{d}\left(\tau_{i}\right)\right)$. Let us introduce an interpolation function $\mu_{i}(\tau)$ which satisfies the following relations:

$$
\begin{aligned}
& \mu_{i}(\tau) \begin{cases}\geq 0, & \tau \in\left[\tau_{i-1}, \tau_{i+1}\right] \\
=1, & \tau=\tau_{i} \\
=0, & \tau \notin\left[\tau_{i-1}, \tau_{i+1}\right]\end{cases} \\
& \mu_{i}(\tau)+\mu_{i+1}(\tau)=1, \quad \tau \in\left[\tau_{i}, \tau_{i+1}\right]
\end{aligned}
$$

Then, an interpolative model over $\tau \in\left[\tau_{i}, \tau_{i+1}\right]$ is given by

$$
\begin{aligned}
\delta \dot{x}(t)= & {\left[\mu_{i}(\tau) A_{i}+\mu_{i+1}(\tau) A_{i+1}\right] \delta x(t) } \\
& +\left[\mu_{i}(\tau) B_{i}+\mu_{i+1}(\tau) B_{i+1}\right] \delta u(t)
\end{aligned}
$$

There are many candidates of the interpolation function $\mu_{i}(\tau)$ which satisfies Eqs. (6a) and (6b). A simple $\mu_{i}(\tau)$ is a linear-type function connecting $i$-th and $(i+1)$-th LTI models by a straight line as shown in Fig. 1.

Using above relations, an interpolative LPV model over $\tau \in\left[0, \tau_{e}\right]$ is given by

$$
\begin{aligned}
\delta \dot{x}(t) & =\sum_{i=1}^{r} \mu_{i}(\tau)\left[A_{i} \delta x(t)+B_{i} \delta u(t)\right] \\
& \triangleq A(\tau) \delta x(t)+B(\tau) \delta u(t)
\end{aligned}
$$




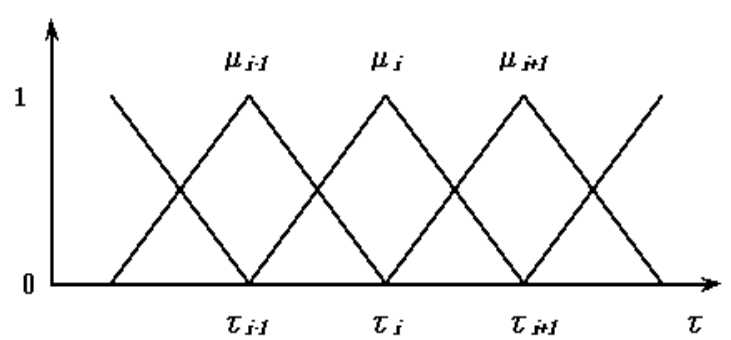

Fig. 1. Linear-type interpolative function.

Another similar modeling is obtained by a T-S fuzzy model in which $\mu_{i}(\tau)$ is given by the fuzzy logic (Palm, et al., 1997; Tang, et al., 1998; Hsieh, et al., 2001). Compared to the T-S model, the time-varying factors in the system are separated from $A$ - and $B$-matrices in Eq. (8). Furthermore, the $i$-th matrices $A_{i}$ and $B_{i}$ are used during $\tau \in\left[\tau_{i-1}, \tau_{i+1}\right]$. Equation (8) is simpler than the T-S fuzzy model.

In construction of T-S model described in (Palm, et al., 1997; Tang, et al., 1998; Hsieh, et al., 2001), the reference trajectory $\left(x^{d}(\tau), u^{d}(\tau)\right)$ is interpolated by fuzzy rules. However, it may be assumed that $\left(x^{d}(\tau), u^{d}(\tau)\right)$ is exactly known because the control objective is tracking of $x(t)$ to $x^{d}(t)$.

\section{DESIGN OF GAIN SCHEDULING}

This section describes a design of a gain scheduling state feedback (GS-SF) law, where the state variable is supposed to be measurable. A GS-SF law for Eq. (8) is given by

$$
\delta u(t)=-F(\tau) \delta x(t)
$$

If the closed-loop matrix $A(\tau)-B(\tau) F(\tau)$ is a stable matrix over the entire operating region, the closedloop system is globally stable. To guarantee the global stability, consider a parameter-dependent Lyapunov function

$$
V(t)=\delta x^{T}(t) P(\tau) \delta x(t), \quad P(\tau)>0
$$

where $P(\tau)$ is a time-varying positive matrix. Then, $d V / d t$ is given by

$$
\frac{d V}{d t}=\dot{x}^{T} P x+x^{T} P \dot{x}+x^{T} \dot{P} x
$$

In this paper, a sufficient condition of the quadratic stability is given by (Fujimori, et al., 1999)

$$
\dot{V}(t)<-x^{T}\left(Q+F^{T} R F\right) x
$$

where $Q \in \Re^{n \times n} \geq 0, R \in \Re^{m \times m}>0$ are weighting matrices and remain to be designed. Letting $X(\tau) \triangleq$
$P^{-1}(\tau)$ and $F(\tau) X(\tau) \triangleq M(\tau)$, inequality (12) is transformed into the following matrix inequality

$$
\begin{aligned}
& \operatorname{He}[A(\tau) X(\tau)-B(\tau) M(\tau)]-\dot{X}(\tau) \\
& \quad+X(\tau) Q X(\tau)+M^{T}(\tau) R M(\tau)<0
\end{aligned}
$$

where $\mathrm{He}[A] \triangleq A+A^{T}$. To express matrix inequality (13) finitely, $M(\tau)$ and $X(\tau)$ are linearly interpolated as

$$
\begin{aligned}
& M(\tau)=\sum_{i=1}^{r} \mu_{i}(\tau) M_{i} \\
& X(\tau)=\sum_{i=1}^{r} \mu_{i}(\tau) X_{i}
\end{aligned}
$$

$\dot{X}$ is approximated as

$$
\dot{X} \simeq \frac{X_{i+1}-X_{i}}{\tau_{i+1}-\tau_{i}}, \quad \tau \in\left[\tau_{i}, \tau_{i+1}\right]
$$

Substituting Eqs. (14), (15) and (16) into Eq. (13) and using the Schur complement (Boyd, et al., 1994), LMIs with respect to $X_{i}$ and $M_{i}(i=1, \cdots, r)$ are derived as follows

$$
\begin{aligned}
& X_{i}>0 \quad(i=1, \cdots, r) \\
& \left(\begin{array}{ccc}
\operatorname{He}\left[A_{i} X_{i}-B_{i} M_{i}\right] & & \star \\
-\frac{X_{j}-X_{i}}{\tau_{j}-\tau_{i}} & \star & \\
H X_{i} & -I_{q} & \star \\
M_{i} & 0 & -R^{-1}
\end{array}\right)<0, \\
& \left(\begin{array}{ccc}
\operatorname{He}\left[A_{j} X_{j}-B_{j} M_{j}\right] & \star & \star \\
-\frac{X_{j}-X_{i}}{\tau_{j}-\tau_{i}} & & \\
H X_{j} & -I_{q} & \star \\
M_{j} & 0 & -R^{-1}
\end{array}\right)<0, \\
& \left(\begin{array}{ccc}
\operatorname{He}\left[A_{i} X_{j}+A_{j} X_{i}\right. & & \\
\left.-B_{i} M_{j}-B_{j} M_{i}\right] & \star & \star \\
-2 \frac{X_{j}-X_{i}}{\tau_{j}-\tau_{i}} & & \\
H\left(X_{i}+X_{j}\right) & -2 I_{q} & \star \\
M_{i}+M_{j} & 0 & -2 R^{-1}
\end{array}\right)<0 \\
& (i=1, \cdots, r-1, \quad j \triangleq i+1)
\end{aligned}
$$

where $\operatorname{rank} Q=q$ and $Q=H^{T} H, H \in \Re^{q \times n}$, ' ‘' means the transpose of the elements located at diagonal position. The number of LMIs to be satisfied is $r+(r-$ 1) $\times 3=4 r-3$. When $X_{i}$ and $M_{i}$ satisfying LMIs (17) and (18) are found, the feedback gain $F(\tau)$ is given by

$$
F(\tau)=\left(\sum_{i=1}^{r} \mu_{i}(\tau) M_{i}\right)\left(\sum_{i=1}^{r} \mu_{i}(\tau) X_{i}\right)^{-1}
$$

\section{SIMULATION RESULT}

This section presents a numerical simulation result where the proposed GS-SF design technique was applied to 
a tracking problem of a two-link robot arm shown in Fig. 2 (Palm, et al., 1997; Hsieh, et al., 2001).

\subsection{Two-link robot arm.}

The masses of the two links were concentrated at the ends and the motor inertia are neglected. The equation of motion of the two-link robot arm was described as

$$
M(x) \dot{x}(t)+N(x)=L u(t)
$$

where $x \triangleq\left[\begin{array}{llll}q_{1} & q_{2} & \dot{q}_{1} & \dot{q}_{2}\end{array}\right]^{T}$ was the state vector including the angles and the angular velocities of the two links, $u \triangleq\left[u_{1}, u_{2}\right]^{T}$ was a torque vector. $M(x)$ included the inertia matrix and $N(x)$ was a vector including centrifugal, Coriolis, gravitational forces and damping. They were given by

$$
\begin{aligned}
& M(x) \\
& =\left(\begin{array}{cccc}
1 & 0 & 0 & 0 \\
0 & 1 & 0 & 0 \\
0 & 0 & \left(m_{1}+m_{2}\right) l_{1}^{2} & m_{2} l_{1} l_{2} \cos \left(q_{1}-q_{2}\right) \\
0 & 0 & m_{2} l_{1} l_{2} \cos \left(q_{1}-q_{2}\right) & m_{2} l_{1}^{2}
\end{array}\right), \\
& N(x)=\left(\begin{array}{c}
-\dot{q} \\
-\dot{q} \\
\frac{m_{2} l_{1} l_{2} \sin \left(q_{1}-q_{2}\right) \cdot \dot{q}}{-\left(m_{1}+m_{2}\right) g l_{1} \sin q_{1}+K_{q_{1}} \dot{q}} \\
\hline-m_{2} l_{1} l_{2} \sin \left(q_{1}-q_{2}\right) \dot{q} \\
-m_{2} g l_{2} \sin q_{2}+K_{q_{2}} \dot{q}
\end{array}\right), \\
& L=\left(\begin{array}{ll}
0 & 0 \\
0 & 0 \\
1 & 0 \\
0 & 1
\end{array}\right)
\end{aligned}
$$

where $m_{i}$ and $l_{i}(i=1,2)$ are respectively the masses and the lengths of the two links. $K_{q_{i}}(i=1,2)$ are the damping coefficients at the hinges.

The control objective was to track the angles and angular velocities of the two links to a given reference trajectory as close as possible. Then, the proposed gain scheduling technique was applied to do this. Numerical values of the two-link robot arm were given as

$$
\begin{aligned}
& m_{1}=1.5 \mathrm{~kg} \quad m_{2}=1 \mathrm{~kg} \\
& l_{1}=0.2 \mathrm{~m} \quad l_{2}=0.2 \mathrm{~m} \\
& K_{q 1}=10 \mathrm{kgm}^{2} / \mathrm{s} \quad K_{q 2}=10 \mathrm{kgm}^{2} / \mathrm{s} \\
& g=9.80165 \mathrm{~m} / \mathrm{s}^{2}
\end{aligned}
$$

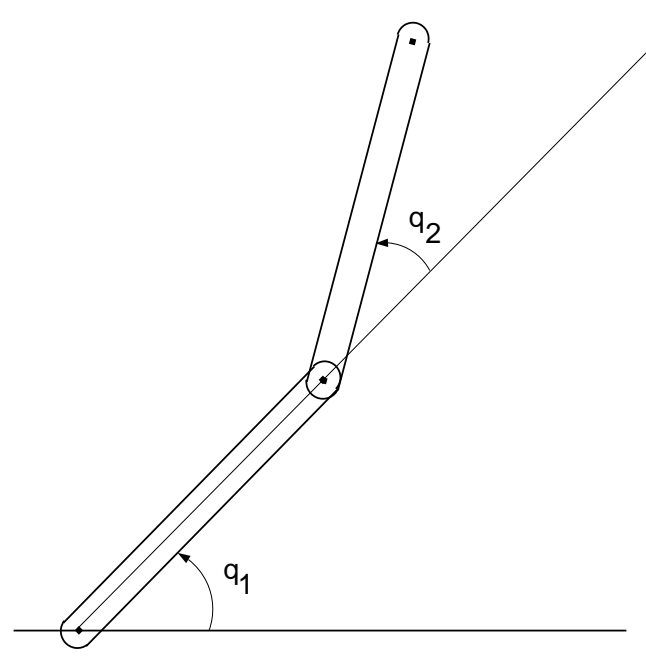

Fig. 2. Two-link robot arm.

A reference trajectory was given by

$$
x^{d}(t)=\left(\begin{array}{c}
0.5-0.5 \sin (\pi t) \\
1.57+0.5 \sin (\pi t) \\
-0.5 \pi \cos (\pi t) \\
0.5 \pi \cos (\pi t)
\end{array}\right)
$$

\subsection{LPV model.}

To derive a linearized equation, $M(x)$ and $N(x)$ were approximated near the reference trajectory as

$$
\begin{aligned}
& M(x) \simeq M\left(x^{d}\right) \\
& N(x) \simeq N\left(x^{d}\right)+\left.\frac{\partial N(x)}{\partial x^{T}}\right|_{x^{d}} \delta x
\end{aligned}
$$

Applying the above approximation to Eq. (20), the linearized equation was obtained as

$$
\begin{aligned}
& \delta \dot{x}(t)=A\left(x^{d}\right) \delta x(t)+B\left(x^{d}\right) \delta u(t) \\
& A\left(x^{d}\right) \triangleq-\left.M^{-1}\left(x^{d}\right) \frac{\partial N(x)}{\partial x^{T}}\right|_{x^{d}}, \\
& B\left(x^{d}\right) \triangleq M^{-1}\left(x^{d}\right) L
\end{aligned}
$$

From Eq. (22), the period of the reference trajectory was $2 \pi / \pi=2 \mathrm{sec}$. Therefore, the range of the operating time was given by $\tau \in[0,2]$. According to Section 2, an interpolative LPV model, named as $P_{l p v}$, was then constructed where the frozen operating times were selected as $\{\tau\}=\{0,0.5,1.0,1.5\}$. For comparison, an LTI model, named as $P_{f i x}$, was obtained at $\tau=1.0$.

To evaluate the model error, the $v$-gap metric was used. The $v$-gap metric is originally introduced to discuss 


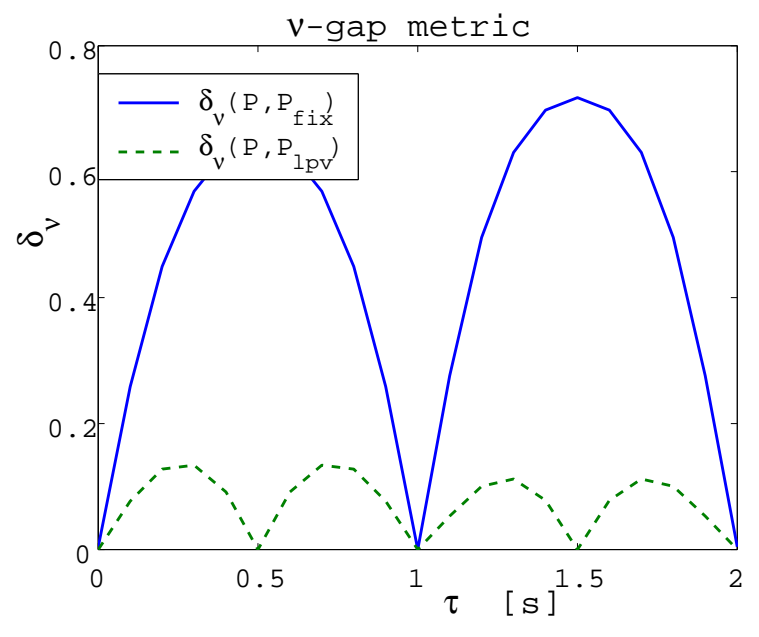

Fig. 3. $\quad v$-gap metric: $\delta_{v}\left(P, P_{f i x}\right)$ and $\delta_{v}\left(P, P_{l p v}\right)$.

the robust stability of LTI systems. It is a measure of uncertainty including the system. In this paper, the $v$ gap metric was used as a measure for evaluating model error of the constructed LPV model. That is, it was desirable that the $v$-gap metric of the LPV model was as small as possible over the operating range. Letting $P_{1}(s)$ and $P_{2}(s)$ be transfer functions of LTI models, the $v$-gap metric between them is defined as follows (Vinnicombe, 2001):

$$
\delta_{v}\left(P_{1}, P_{2}\right) \triangleq \sup _{\omega} \kappa\left(P_{1}(j \omega), P_{2}(j \omega)\right)
$$

where

$$
\kappa(X, Y) \triangleq \bar{\sigma}\left[\left(I+Y Y^{*}\right)^{1 / 2}(Y-X)\left(I+X X^{*}\right)^{1 / 2}\right]
$$

where $\bar{\sigma}[\cdot]$ means the maximum singular value. The range of $\delta_{v}\left(P_{1}, P_{2}\right)$ is $\delta_{v} \in[0,1]$. The larger $\delta_{v}$ is, the further $P_{1}$ is from another model $P_{2}$. Using the $v$-gap metric, the model error of an interpolative LPV model is evaluated with respect to the operating time.

Figures 3 shows the plots of the $v$-gap metric $\delta_{v}\left(P, P_{f i x}\right)$ and $\delta_{v}\left(P, P_{l p v}\right)$ with respect to the operating time $\tau$ where $P(s ; \tau)$ is a transfer function of an LTI model which is exactly derived at $\tau$. The $v$-gap metric was increased when $\tau$ was shifted from the selected frozen operating times. By increasing the number of the selected frozen operating times $r$, the maximum value and the area of $\delta_{v}\left(P, P_{l p v}\right)$ over $\tau \in[0,2]$ were reduced.

\subsection{GS control.}

A GS-SF law was designed with $P_{l p v}$ by the proposed technique. For comparison, a fixed state feedback (FixSF) law was designed with $P_{\text {fix }}$ by the LQR technique.

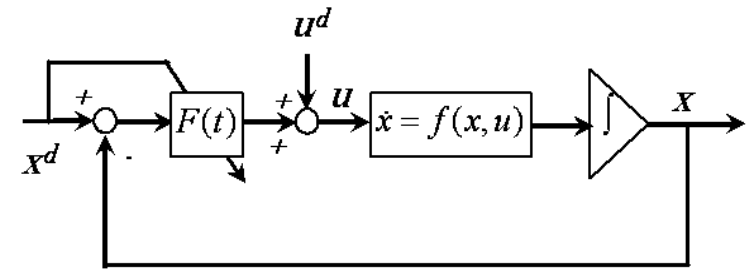

Fig. 4. Control system of two-link robot arm using GS-SF.

The weighting matrices in Eq. (12) was given by

$$
\begin{aligned}
& Q=H^{T} H, \quad R=I_{2} \\
& H=\left(\begin{array}{cccc}
100 & 0 & 0 & 0 \\
0 & 100 & 0 & 0
\end{array}\right)
\end{aligned}
$$

Figure 4 shows a block diagram of the control system where the GS-SF law was implemented. When operating the system, $\tau$ was synchronized with $t$. The input to the two-link robot arm was given by

$$
u(t)=-F(t)\left[x(t)-x^{d}(t)\right]+u^{d}(t)
$$

In the GS-SF, the state feedback gain $F(t)$ was changed with respect to $t$, while in the Fix-SF, the gain was constant.

Figures 5 and 6 show the plots of tracking errors; that is, the time responses of the deviation state variable $\delta x(t)=x(t)-x^{d}(t)$ in the closed-loop system where both state feedback laws were applied to the nonlinear equation (20) as shown in Fig. 4. The angles of the two-link robot arm $x_{1}(t)=q_{1}(t)$ and $x_{2}(t)=q_{2}(t)$ converged to their references, while the angular velocities $x_{3}(t)=\dot{q}_{1}(t)$ and $x_{4}(t)=\dot{q}_{2}(t)$ deviated from the references. The responses of $q_{1}(t)$ and $q_{2}(t)$ by the Fix-SF were faster than those by the GS-SF, but an oscillation was cyclically observed in the responses of $x_{3}(t)$ and $x_{4}(t)$ by the Fix-SF law. It is concluded from these figures that the constructed LPV model $P_{l p v}$ appropriately expressed the behavior of the nonlinear equation (20) along the reference trajectory. The GS-SF law designed using the LPV showed better tracking performance than the Fix-SF law.

\section{CONCLUDING REMARKS}

This paper has presented a gain scheduling control of a nonlinear system in which the reference trajectory was given in advance. Multiple frozen operating times were chosen on the reference trajectory and an LTI 

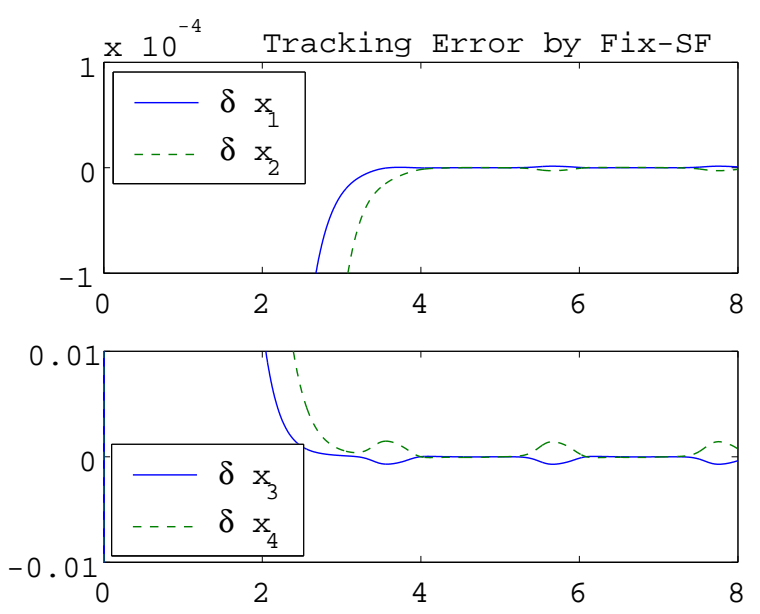

Fig. 5. Tracking error by Fix-SF.

model was obtained at each operating time. An LPV model was then constructed by interpolating the region between the neighbor frozen operating times. A GSSF law was designed by an LMI formulation. The effectiveness was demonstrated in a numerical simulation of a tracking control of a two-link robot arm. This paper has not mentioned how multiple operating times should be selected. Fujimori et al. (Fujimori, et al., 2004) proposed a method for selecting the frozen operating times to minimize the interpolative error of the constructed LPV model.

\section{REFERENCES}

Boyd, S., L. El Ghaoui, E. Feron and V. Balakrishnan (1994). Linear Matrix Inequalities in System and Control Theory, SIAM Studies in Applied Mathematics 15, SIAM, Philadelphia.

Fujimori, A., H. Tsunetomo and Z.-Y. Wu (1999). "Gain-scheduled control using fuzzy logic and its application to flight control," AIAA J. Guidance, Control and Dynamics, 22, 175-178.

Fujimori, A., F. Terui and P.N. Nikiforuk (2004). "Flight control design of an unmanned space vehicle using gain scheduling," AIAA J. Guidance, Control and Dynamics, 27.

Hsieh, C.-H., J.-H. Chou and Y.-J. Wu (2001). “Optimal Takagi-Sugeno fuzzy gain-scheduler design using Taguchi-MHGA method," JSME Int. J. Series $C, \mathbf{4 4}, 143-155$.

Leith, D.J. and W.E. Leithead (2000). "Survey of gainscheduling analysis and design", Int. J. Control, 73, 1001-1025.
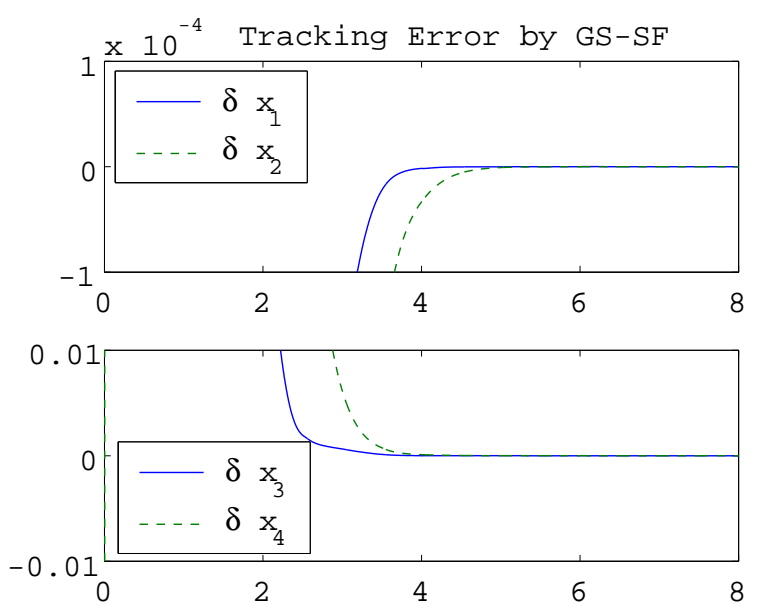

Fig. 6. Tracking error by GS-SF.

McFarlane, D.C. and K. Glover (1990). Robust Controller Design Using Normalized Coprime Factor Plant Descriptions, Lecture Notes in Control and Information Sciences, No. 138, Springer-Verlag, Berlin.

Palm, R., D. Driankov and H. Hellendoorn (1997). Model Based Fuzzy Control, Springer-Verlag, Berlin.

Rugh, W.J. and F. Shamma (2000). "Research on Gain Scheduling," Automatica, 36, 1401-1425.

Takagi, T. and M. Sugeno (1985)., "Fuzzy identification of systems and its applications to modeling and control," IEEE Trans. on Sys., Man and Cybernet., 15, 116-132.

Tang, K.S., K.F. Man, Z.F. Liu and S. Kwong (1998). "Minimal fuzzy memberships and rules using hierarchical genetic algorithm," IEEE Trans. on Industrial Electronics, 45, 162-169.

Vinnicombe, G. (2001). Uncertainty and Feedback $\left(\mathrm{H}_{\infty}\right.$ loop-shaping and the $v$-gap metric), Imperial College Press, London.

Zhou, K., J.C. Doyle and K. Glover (1996). Robust and Optimal Control, Prentice-Hall, Englewood Cliffs, NJ. 